\title{
An analytic description of thick-wall bubbles*
}

\author{
Jooyoo Hong \\ Department of Physics \\ University of Florida \\ Gainesville, FL 32611
}

JUN 301992

\begin{abstract}
A new approximation scheme to the false-vacuum decay is suggested. In this scheme the bounce solutions can be obtained in an explicit and analytic way even for thick-wall bubbles. The result is compared with Coleman's thin-wall description, which shows that it nicely comprises the result of the latter prescription. Some applications are also discussed.
\end{abstract}

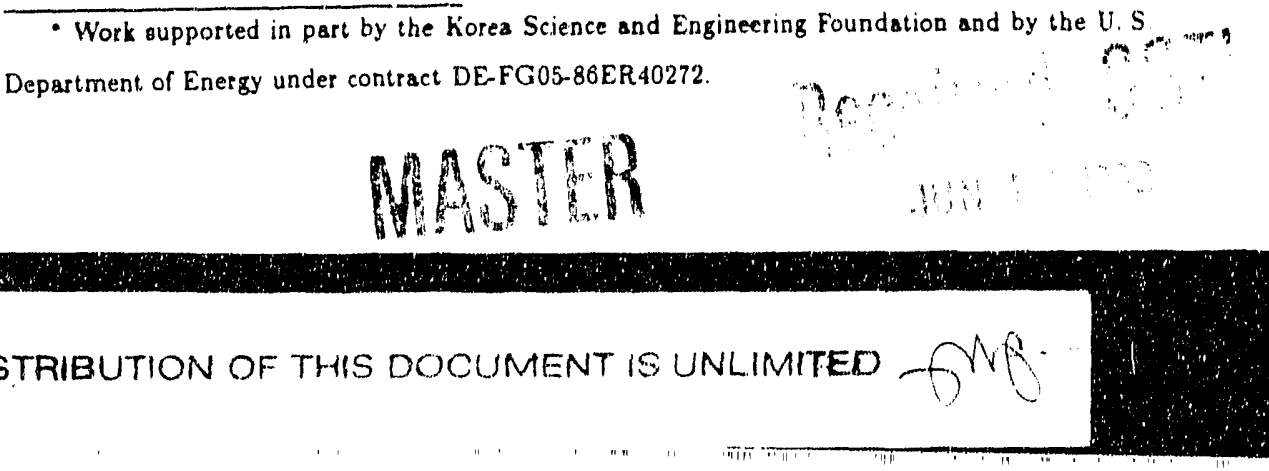


Phase transitions have been discussed in innumerable contexts of physics. Among them, vacu:m phase transitions in the cosmological settings have profound implications for both cosmology and elementary particle physics. They are the turning points in the history of our universe. The characters of the phase transitions can play decisive roles in the subsequent evolution of the universe. While the second-order transitions are usually smooth. the first-order transitions are dramatic and bring forth many interesting questions. In the discussion of first-order phase transitions the most important ingredient is the calculation of the false-vacuum decay rates. Coleman[1] laid a groundwork on this matter. Even though he discussed false-vacuum decays in a broader sense, one of his main results is the so-called thin-wall approximation to the vacuum transition betwcen two nearly degenerate vacua. ${ }^{2}$ He considered a special, but generic, model of a single scalar with a metastable minimum. For this case he could get analytic expressions for thin-wall bubble solutions and the corresponding actions, assuming $O(4)$ symmetry and the near degeneracy of the minima. However, the latter assumption of near degeneracy is not satisfied for most of the parameter range in the models of our interests, e.g. SU(5) GUT models[3]. So, for more realistic (and thus interesting) cases we should consider thick-uall solutions. One way to study this is to perform a humid numerical calculation of obtaining a bounce solution and computing its action. This is what people were actually doing. But the involved amount of numerical work is daunting. And without an explicit analytic solution it would not be easy to get an insight into the nature of first-order phase transitions emerging from various models. What we do in this paper is a try to obtain an approximate but useful analytic expression for a generic thick-wall bubble.

Let us consider a simple model that gives a first-order phase transition. A necessary condition for this model is that it has at least one metastable minimum as well as a global minimum. For this model to be generic, we should be able to vary independently the height of the energy difference between the metastable point and the global minimum, the distance between the two vacua, and the height of the potential barrier. The essential features of the bounce solution are mostly determined

${ }^{2}$ The validity of the thin-wall approximation was questioned in Ref. [2]. 
from these informations only. Then the simplest model with these properties is none other than a single scalar model of which Lagrangian is written as

$$
\mathcal{L}=\frac{1}{2} \partial_{\mu} \phi \partial^{\mu} \phi-V(\phi)
$$

where

$$
V(\phi)=\phi^{2}\left(a \phi^{2}+b v \phi+c v^{2}\right)
$$

with the constraints $\delta V / \delta \phi=0$ at $\phi=v$, i.e. $4 a+3 b+2 c=0$ and $V(v)<0$, i.e. $a+b+c<0$. This is also the only renormalizable one among the possible choice of potentials. Assuming $O(4)$ symmetry, the euclideanized action and the equation of motion are

$$
S_{E}=2 \pi^{2} \int_{0}^{\infty} r^{3} d r\left[\frac{1}{2}\left(\frac{d \phi}{d r}\right)^{2}+V(\phi)\right]
$$

and

$$
\frac{d^{2} \phi}{d r^{2}}+\frac{3}{r} \frac{d \phi}{d r}=\frac{\delta V}{\delta \phi}
$$

where $r=\left(x_{\mu} x_{\mu}\right)^{1 / 2}$. For convenience we rescale all the dimensionful quantities appropriately to make them dimensionless: $\sigma=\phi / v, U=V / a v^{4}$, and $x=\sqrt{2 a} v r$. Then the above equations are written in terms of the rescaled quantities as

$$
S_{E}=\frac{\pi^{2}}{a} \int_{0}^{\infty} x^{3} d x\left[\frac{1}{2}\left(\frac{d \sigma}{d x}\right)^{2}+\frac{1}{2} U(\sigma)\right]
$$

and

$$
\frac{d^{2} \sigma}{d x^{2}}+\frac{3}{x} \frac{d \sigma}{d x}=\frac{1}{2} \frac{\delta U}{\delta \sigma}
$$

Redefine the parameters as $c / a=\kappa$ (thus $b / a=-2(\kappa+2) / 3$ ), then the potential $U(\sigma)$ becomes

$$
U(\sigma)=\sigma^{2}\left[\sigma^{2}-\frac{2}{3}(\kappa+2) \sigma+\kappa\right]
$$

Our choice of $\sigma=0$ as a metastable vacuum and of $\sigma=1$ as a true vacuum restricts $\kappa$ to be $0<\kappa<1$. At $\kappa=0$ the barrier between two vacua disappears and at $\kappa=1$ these two vacua become degenerate. The potential looks like as in Fig. 1. In order to compute the action, first we should be able to get the bounce solution $\sigma(x)$. However, with the inclusion of the friction term the equation will never be integrable. Even 
the boundary value of $\sigma$ at $x=0$ denoted by $\sigma_{0}$ cannot be known until we get a full solution. For any nontrivial potential the only way to get an exact solution seems to be a numerical one. So we will disregard the friction term, but as a compensation deform the potential $U(\sigma)$ to $\dot{U}(\sigma)=U(\sigma)+\Delta U(\sigma)$.

$$
\frac{d^{2} \sigma}{d x^{2}}=\frac{1}{2} \frac{\delta \check{U}}{\delta \sigma}
$$

where $\dot{U}(0)=0$. In principle one can always find this modified potential that shares the same solution with the original equation if we do not restrict $\tilde{U}(\sigma)$ to some particular form. However, for the integrability we will sacrifice this freedom by restricting the form of the deformation as $\Delta U(\sigma)=\alpha \sigma^{4}+\beta \sigma^{3}+\gamma \sigma^{2}(\alpha, \beta$, and $\gamma$ are deformation parameters), which still renders $\tilde{U}$ renormalizable. This, certainly, would result in the deviation from the actual solution. But, if the deviation is not large, this restriction would be worthy of doing in light of integrability. The deformed potential is then given by

$$
\tilde{U}(\sigma)=A \sigma^{2}\left(\sigma^{2}-B \sigma+C^{2}\right)
$$

where $A \equiv 1+\alpha, B \equiv(-\beta+2(\kappa+2) / 3) / A$, and $C \equiv((\kappa+\gamma) / A)^{1 / 2}$. Since the core value of the bounce solution, i.e. $\sigma_{0}$, is neither the point where $U\left(\sigma_{0}\right)=0$ nor $\sigma_{0}=1$ (the true vacuum) but is somewhere between these two points, we deform the original potential to set $\check{U}\left(\sigma_{0}\right)=0$. This should be the case because we are neglecting the friction. Thus, there follows a relation

$$
\sigma_{0}^{2}-B \sigma_{0}+C^{2}=0
$$

Now it is easy to integrate Eq. (8). Using the relation in Eq. (10), we obtain the following solution

$$
\sigma(z)=\sigma_{0}\left(\cosh ^{2} \frac{C z}{2}-\frac{\sigma_{0}^{2}}{C^{2}} \sinh ^{2} \frac{C z}{2}\right)^{-1}
$$

where $z \equiv \sqrt{A} x$.

From this explicit bubble solution we can find the width of the bubble wall $\Delta$ and the radius of the bubble $k$. Obviously the width of the bubble wall is given by

$$
\Delta \simeq 1 /(C \sqrt{2 a A v})
$$


And defining the location of the wall as the point where $\sigma$ reaches half ol its core value, i.e. $\sigma_{0} / 2$, we find

$$
R \simeq \Delta \cdot \cosh ^{-1}\left(\frac{3 C^{2}-\sigma_{0}^{2}}{C^{2}-\sigma_{0}^{2}}\right)
$$

Certainly $\sigma_{0}, A, B$, and $C$ are parameters that should be determined in terms of $r$. We can determine these by matching the derivatives of $\sigma$ with respect to $x$ at origin from Eq. (6) and from Eq. (8) respectively. Since we are left with three unknown parameters ( $B$ is determined from Eq. (10)), we need to match three different derivatives to get three independent relations. But, in this way we end up with very complicated expressions for $\sigma_{0}, A$, and $C$ in terms of $\kappa$, which is not desirable. So we simplify the problem by setting $A=1$. And we also determine $\sigma_{0}$ empirically using the numerical result. We then need only one derivative matching: ${ }^{3}$

$$
\begin{array}{ll}
\sigma(x)=\sigma_{0}-\frac{1}{4} \sigma_{0}\left(\sigma_{0}-\frac{\kappa}{2}\right)\left(1-\sigma_{0}\right) x^{2}-\cdots & \text { (from Eq. (6)) } \\
\sigma(x)=\sigma_{0}-\frac{1}{4} \sigma_{0}\left(C^{2}-\sigma_{0}^{2}\right) x^{2}-\cdots & \text { (from Eq. (8)) }
\end{array}
$$

Through this procedure we arrive at another relation

$$
C^{2}=\left(1+\frac{\kappa}{2}\right) \sigma_{0}-\frac{\kappa}{2}
$$

Using the numerical result, we find an empirical (not derived) form"la for $\sigma_{0}$

$$
\sigma_{0}=1-\exp \left(-\frac{\kappa(4-\kappa)}{1-\kappa}\right) .
$$

For comparison we plot the above expression and the numerical result in Fig. 2. 'The formula reproduces the actual value within an accuracy about $1 \%$. And thus, from Eq. (14),

$$
C^{2}=1-\left(1+\frac{\kappa}{2}\right) \exp \left(-\frac{\kappa(4-\kappa)}{1-\kappa}\right)
$$

When $\kappa$ goes to 1 (thus both $\sigma_{0}$ and $C$ goes to 1 ), the solution describes a thin-wall bubble, i.e. $R / \Delta \gg 1$. To be more quantitative, when $\delta \equiv 1-\kappa \ll 1$,

\footnotetext{
${ }^{3}$ Because of a (unphysical) refection symmetry of the equations of motion under $x \rightarrow-x, \sigma(x)$ is an even function of $x$.
} 


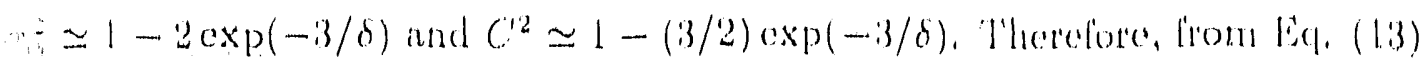

$$
\frac{R}{\Delta} \simeq \frac{3}{\delta} \gg 1
$$

And in this case the solution becomes

$$
\sigma(x) \simeq \begin{cases}1 & x \ll R / \Delta \\ \frac{1}{2}\left[1-\tanh \left(x-\frac{R}{\Delta}\right)\right] & x \simeq R / \Delta \\ 0 & x \gg R / \Delta .\end{cases}
$$

Howerer, as $r$ goes away from 1 , it becomes a thick-wall bubble. In particular, when $n$ is very small, $\sigma_{0} \simeq 4 \kappa$ and $C \simeq \sqrt{7 \kappa / 2}$. Thus, $R / \Delta \simeq O(1)$. In this limit the solution becomes

$$
\sigma(x) \simeq \frac{4 \kappa}{\cosh ^{2}(\sqrt{7 \kappa / 8} x)}
$$

Even though it is hardly possible to get a simple formula for $S_{E}$ applicable for the entire range of $k$, we are ready to compute it at least for the aforementioned two interesting limits. First, in the thin-wall limit, using the solution in Eq. (18),

$$
\begin{aligned}
S_{E} & =\frac{\pi^{2}}{2 a} \int_{0}^{\infty} d x x^{3}\left[\left(\frac{d \sigma}{d x}\right)^{2}+U(\sigma)\right] \\
& \simeq \frac{\pi^{2}}{2 a}\left[\left(\frac{3}{\delta}\right)^{3} \cdot \frac{1}{3}-\frac{\delta}{3} \cdot \frac{1}{4}\left(\frac{3}{\delta}\right)^{4}\right] \\
& =\frac{9 \pi^{2}}{8 a \delta^{3}}
\end{aligned}
$$

where $\delta=1-\kappa$. This is exactly the same formula with that obtained in the conventional nalysis, ${ }^{4}$ which assures that we are on the right track. Likewise, in the small $k$ lin it (the case of shallow barrier, namely the case of weakly first-order phase transition we use Eq. (19) to get the action

$$
S_{E}=\frac{2.59 \pi^{2}}{a} \kappa+O\left(\kappa^{2}\right)
$$

\footnotetext{
${ }^{4}$ For exam $)$ le, in page 201 of Ref. [4], translating into our notations by replacing $\lambda$ by $4 a$ and $\epsilon$ by $\delta / 6$, we see it reproduces the same formula in Eq. (20).
} 


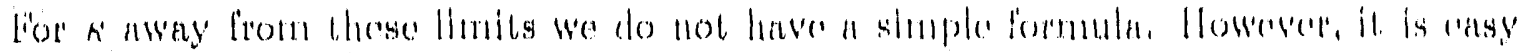

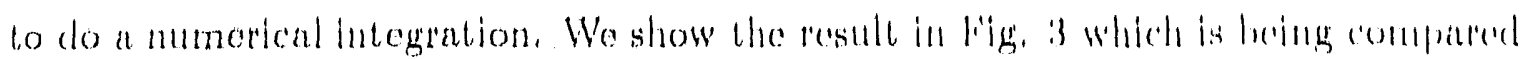

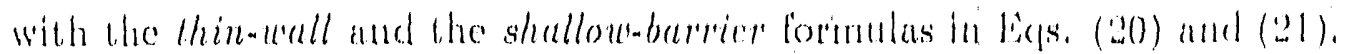

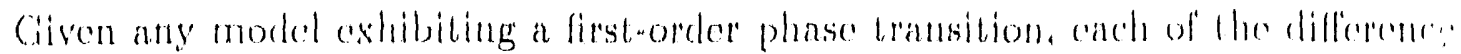
between vactum energy densities, the distance between two sacha, and the herght of the potential barrier set equal to a corresponding quantity in the real scalar model that we are discussing:

$$
\begin{array}{ll}
\text { distance between two vacua } & \Longrightarrow \\
\text { energy-density difference } & \Longrightarrow \frac{1}{3} a v^{4}(1-n) \\
\text { barrier height } & \Longrightarrow \frac{1}{48} a v^{4} k^{3}(4-n)
\end{array}
$$

Now we can write $a, v$, and $\kappa$ in terms of the parameters of this particular model. Then we know the (approximate) shape of the bubble solution and its corresponding action without cumbersome analysis.

In conclusion, we obtained an analytic expression for a bubble solution which holds for a thick-wall bubble as well as for a thin-wall bubble. Our solution nicely comprises Coleman's thin-wall solution as a special limit.

\section{Acknowledgements}

I. would like to thank S.-J. Sin for discussions. I appreciate the hospitality of the Center for Theoretical Physics (Seoul National University) during the visit.

\section{DISCLAIMER}

This report was prepared as an account of work sponsored by an agency of the United States Government. Neither the United States Government nor any agency thereof, nor any of their employees, makes any warranty, express or implied, or assumes any legal liability or responsibility for the accuracy, completeness, or usefulness of any information, apparatus, product, or process disclosed, or represents that its use would not infringe privately owned rights. Reference herein to any specific commercial product, process, or service by trade name, trademark, manufacturer, or otherwise does not necessarily constitute or imply its endorsement, recommendatiun, or favoring by the United States Government or any agency thereof. The views and opinions of authors expressed herein do not necessarily state or reflect those of the United States Government or any agency thereof. 


\section{References}

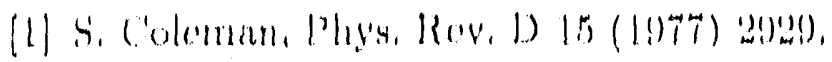

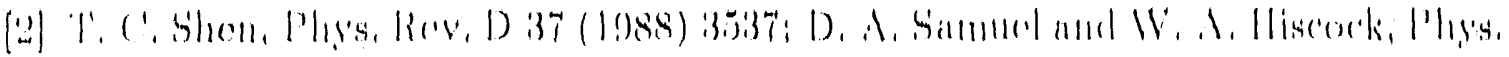
l.el1. $132(6)(1991) 2351$.

[3] A. II, Cinth and E. J, Weinbers, Phys, Rev, D 23 (1981) 876.

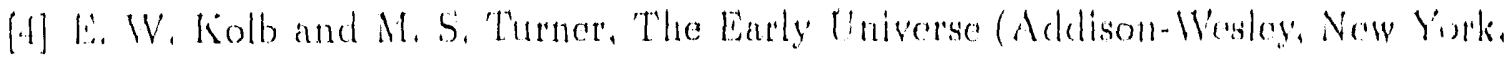
1.90()). 


\section{litgure Captions}

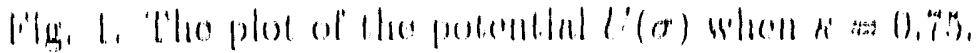

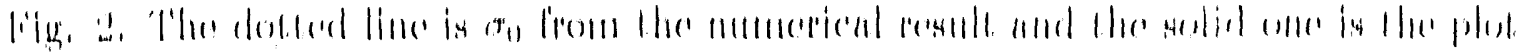
oflity. ilit).

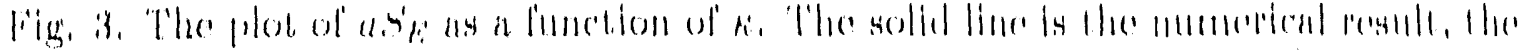

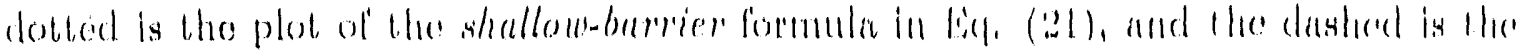
Hhin-urall action in Eq. (20). 
1

1

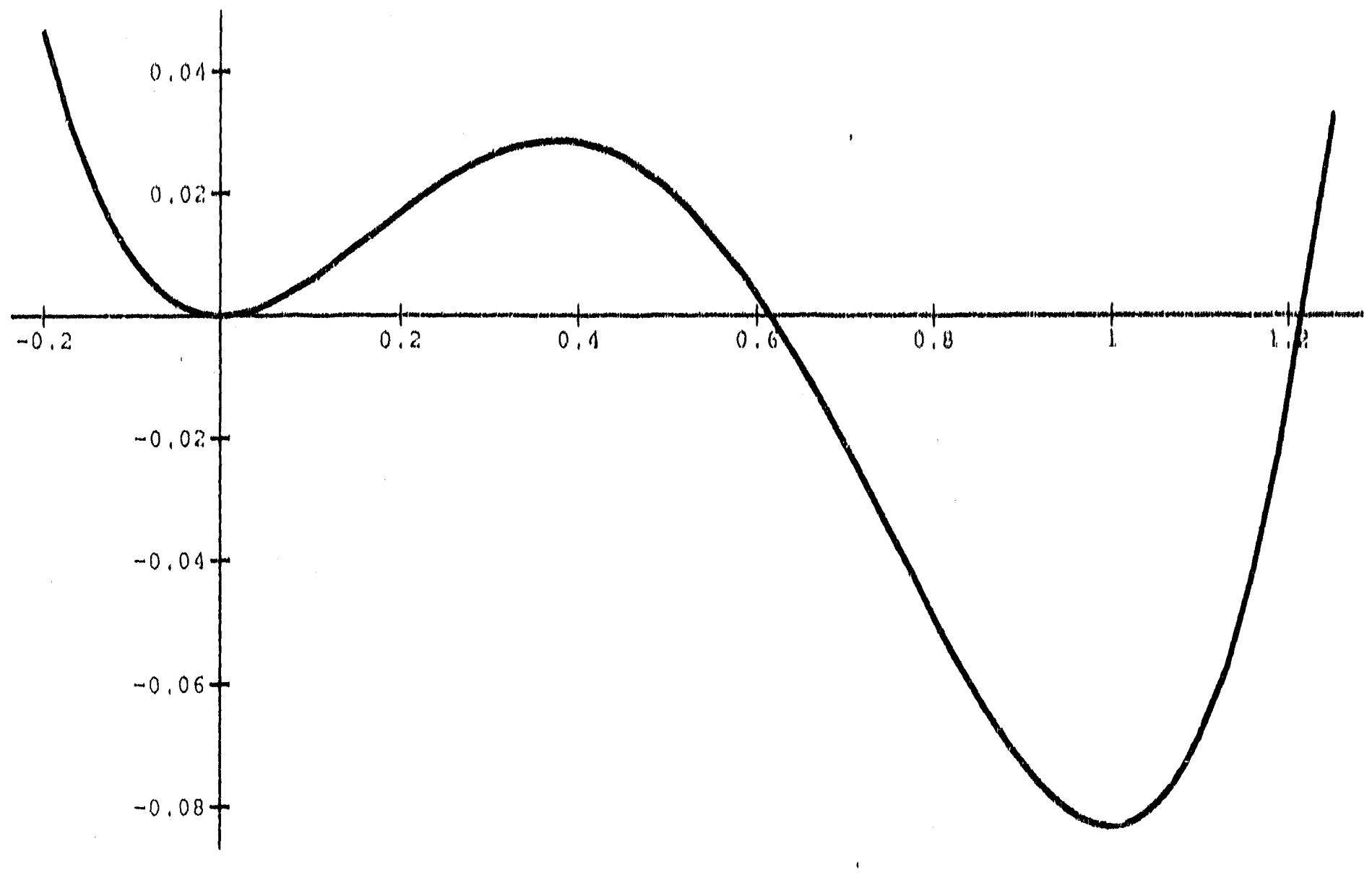

Fig. 1 
stom 0

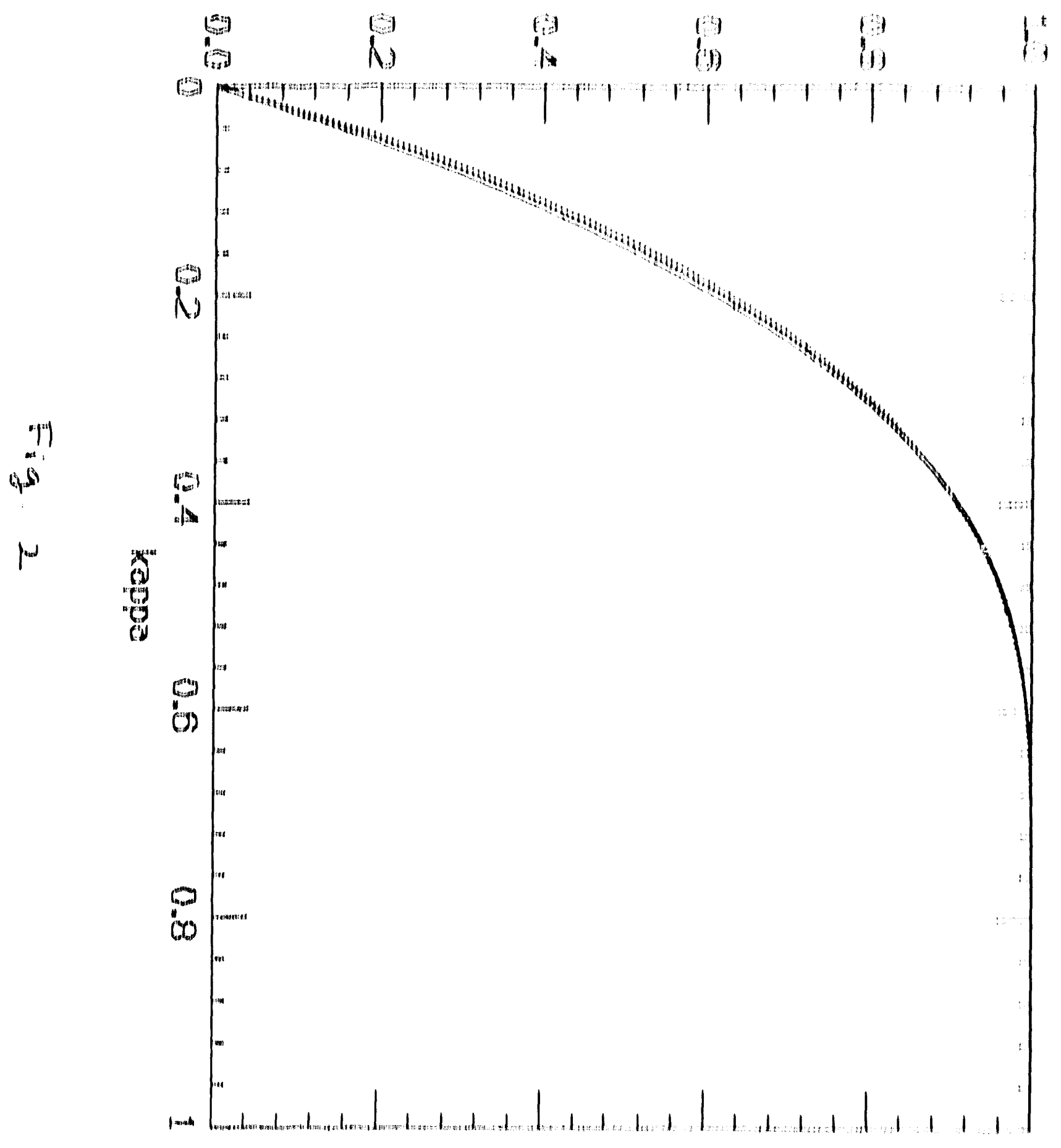




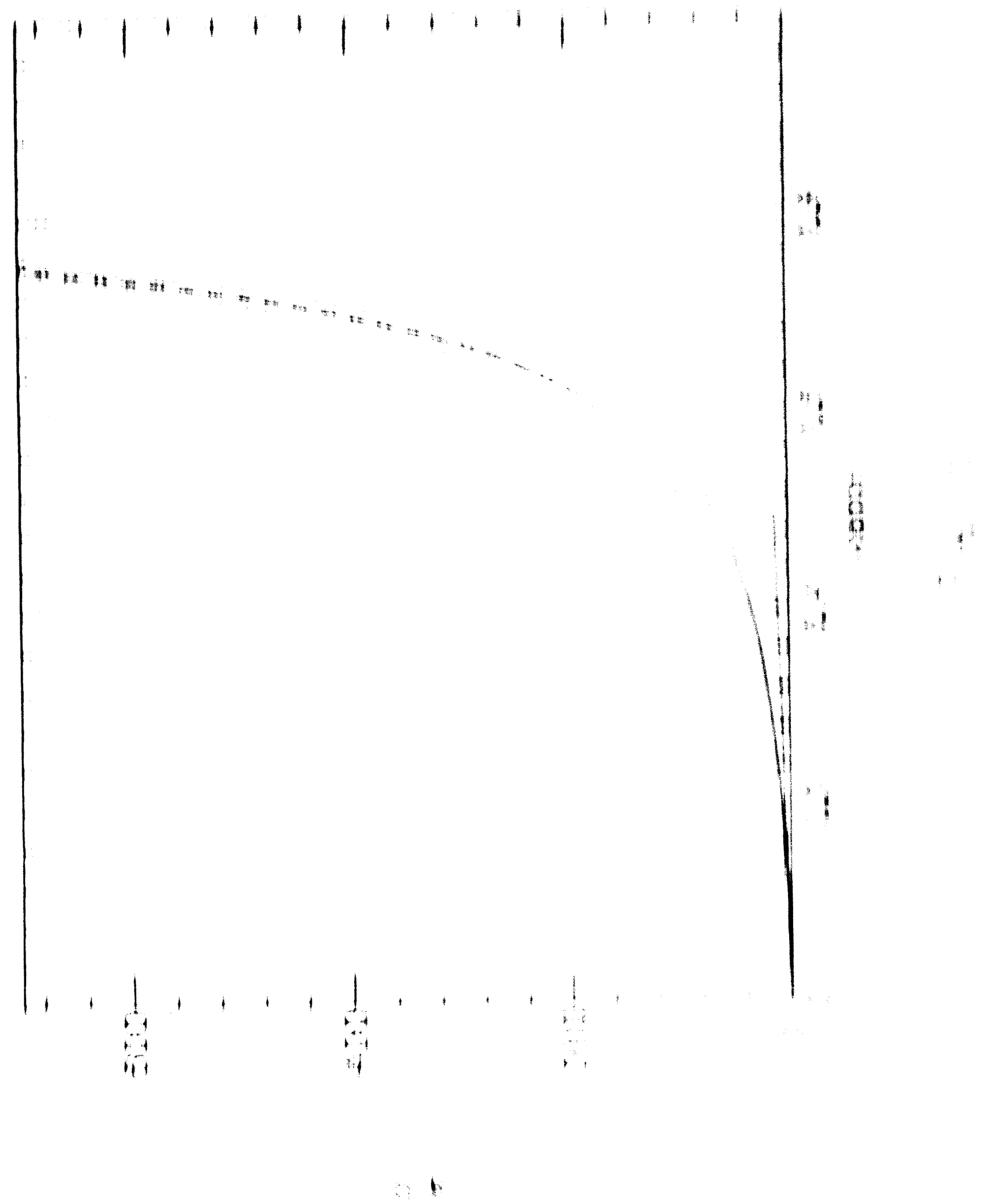




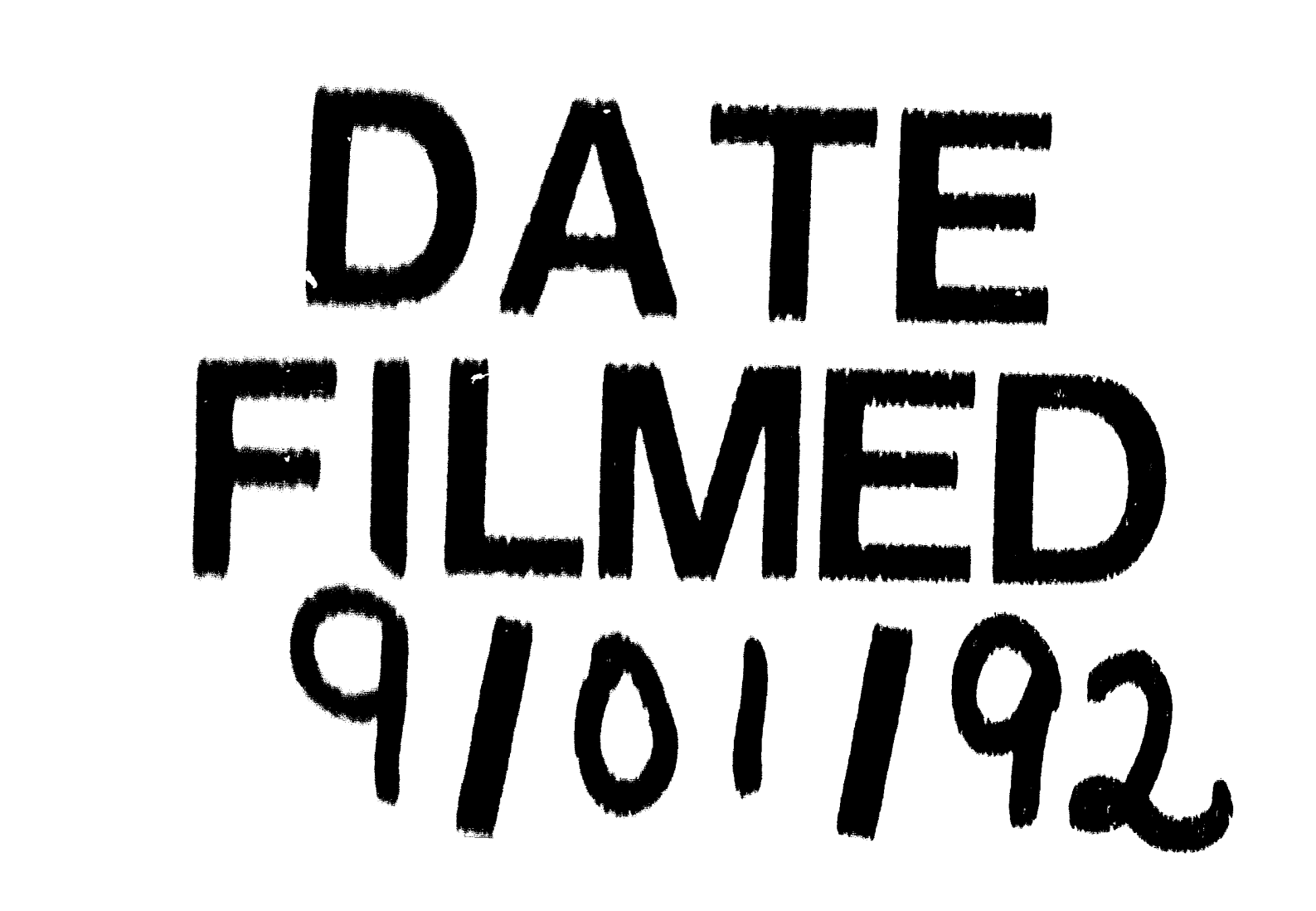


